\title{
APROXIMACIÓN A LA VEGETACIÓN ALGAL DOMINADA POR LAURENCIA PAPILLOSA (C. AGARDH) GREVILLE, EN LA ZONA MEDIOLITORAL DEL SURESTE DE ESPAÑA
}

\author{
Juan SOTO MORENO y Francisco CONDE POYALES
}

\begin{abstract}
RESUMEN. Aproximación a la vegetación algal dominada por Laurencia papillosa (C. Agardh) Greville, en la zona mediolitoral del Sureste de España. Se estudia la comunidad de la zona mediolitoral inferior constituida por Laurencia papillosa (C. Agardh) Greville en base a 21 inventarios realizados durante un ciclo anual en las costas de Murcia y Almería. Se constata una alta riqueza florística con una estratificación algal en cinco niveles así como la existencia de una dominancia neta de las algas mediolitorales e infralitorales fotófilas sobre otros grupos ecológicos, un elevado grado de equitabilidad específica y una estacionalidad netamente marcada. Finalmente se postulan argumentos que individualizan esta comunidad de otras de la zona mediolitoral inferior del Mediterráneo Occidental.
\end{abstract}

Palabras clave. Vegetación, Laurencia papillosa, algas, Sureste de España.

SUMMARY. A study of Laurencia papillosa (C. Agardh) Greville community in the SE of Spain. The intertidal community of Laurencia papillosa (C. Agardh) Greville has been studied by means of 21 inventories made along an annual cycle (Murcia and Almería). The community has a rich flora which is dominated by intertidal an sublittoral photophilic algae, a high degree of specific equitability and patent seasonal changes. The constitution of Laurencia papillosa community seems to be specifically different from other intertidal phytobenthic communities from the Western Mediterranean.

Key words. Vegetation, Laurencia papillosa, algae, South-East of Spain.

\section{INTRODUCCIÓN}

Laurencia papillosa (C. Agardh) Greville es una rodomelácea de presencia usual en la zona mediolitoral inferior de la cuenca mediterránea (Mayhoub, 1976: Ballesteros y Romero, 1982; Ribera y Gómez Garreta, 1984; Giaccone et al., 1985; Boudouresque \& Perret, 1987) aunque sólo forma poblaciones homogéneas en sus áreas más cálidas.
En el litoral mediterráneo español ha sido citada en Cataluña (Ballesteros y Romero, 1982) aunque de forma aislada y dispersa (Ballesteros com. pers.), litoral valenciano (abundante en la provincia de Alicante: Barceló, 1987), Islas Baleares (Ribera y Gómez Garreta, 1984), litoral murciano almeriense (Soto, 1987) y se carece de reseñas de ella en los litorales de Granada (Varo et al., 1979; Conde y Soto, 1986 y 1987; Soto, 1991), Málaga (Conde, 
1984) y Cádiz (Seoane, 1965). Constituye horizontes de vegetación en las Islas Baleares más meridionales y en el Sur del País valenciano (Terradas, 1989). En ninguna de las áreas citadas existen datos sobre la comunidad que define.

Dada la importancia fisionómica del horizonte caracterizado por Laurencia papillosa en el Sureste de España, se han realizado una serie de inventarios florísticos en varios puntos del litoral murciano-almeriense donde el horizonte aparece claramente definido, con objeto de poder determinar su composición florística, estratificación vertical, cambios estacionales en composición florística, grupos ecológicos mejor representados y su evolución anual, estructura a pequeña escala y evolución anual de sus parámetros estructurales.

\section{MATERIAL Y MÉTODOS}

El material estudiado se recolectó durante el año 1985 en cuatro localidades del Sureste de España (provincias de Murcia y Almería): A: Calnegre (Murcia) (enero, marzo, mayo y octubre), C: Calarreona (Murcia) (enero, marzo, junio, agosto septiembre, octubre, noviembre y diciembre), D: Agua Amarga (Almería) (febrero, marzo, julio y diciembre) y B: Monsul-Cabo de Gata (Almería) (febrero, marzo, julio, octubre y diciembre). La superficie inventariada ha sido siempre superior a $225 \mathrm{~cm}^{2}$ (cuadrado de 15 por $15 \mathrm{~cm}$ ), suficiente para disponer de una buena representación de la flora y estructura de las comunidades mediolitorales (Boudouresque, 1974; Cinelli et al., 1977; Coppejans, 1980; Ballesteros, 1984). Las muestras siempre se obtuvieron por separación total junto al sustrato rocoso, con objeto de disponer también de representación de la flora del estrato incrustante.

Para el análisis de los inventarios se ha consultado las siguientes fuentes bibliográficas que se relacionan por orden cronológico:
De Boudouresque (1971 a) se ha utilizado los siguientes parámetros:

a)Recubrimiento: Medida de la superficie $\left(\right.$ en $\left.\mathrm{cm}^{2}\right)$ obtenida por proyección del talo abatido de la especie sobre el plano horizontal.

b) Dominancia cuantitativa (o en función del recubrimiento): cociente, expresado porcentualmente, entre el recubrimiento de una especie o grupo de éstas y la suma del recubrimiento de todas las especies del inventario.

c) Tensión: cociente entre el porcentaje de especies de un grupo y su recubrimiento global. Se ha interpretado exclusivamente como índice de relevancia fisionómica.

De Boudouresque (1984) se ha utilizado el siguiente parámetro:

a) Taxonomía de grupos ecológicos del Mediterráneo noroccidental, pero incluyendo o segregando, en algún caso, taxones que muestran, claramente, diferente ubicación ecológica en esta área geográfica.

De Ballesteros (1984 y 1986) se han utilizado parámetros para el estudio de la estructura a pequeña escala de la comunidad. Previamente cada muestra se ha subdividido en submuestras de $9 \mathrm{~cm}^{2}$. Los parámetros estructurales se han obtenido de dos tipos de ajustes matemáticos a curvas experimentales: la curva experimental número medio de especies/área se ha ajustado a una función semilogarítmica y la curva experimental diversidad media/área a una función de Michaelis-Menten. A partir de estas funciones matemáticas teóricas obtenidas se han calculado los parámetros utilizados en el estudio estructural (siempre que sus coeficientes de correlación $-\mathrm{r}^{2}$-fuesen superiores a 0.75$)$ que se relacionan:

a) Parámetros derivados de la función semilogarítmica:

a-1)Area mínima cualitativa: subjetivamente se ha considerado suficientemente representativa aquella que incrementada en un $20 \%$ no genera incrementos florísticos superiores al $5 \%$. 
a-2) Riqueza específica: subjetivamente se ha considerado el número de taxones que coincide con una pendiente de la función semilogarítmica de 0.05 (Punto Calleja).

b) Parámetros derivados de la función de Michaelis-Menten:

b-1) Estima de la alfa-diversidad como indicadora de equitabilidad.

b-2) Estima de la beta-diversidad como indicadora de la forma del espectro de diversidad y consecuentemente del nivel de organización estructural de la comunidad.

b-3) Area mínima estructural: subjetivamente se ha considerado el área que coincide con una pendiente de 0.001 de la función de Michaelis-Menten.

Los resultados del análisis de los inventarios se han agrupado en periodos bimensuales para una mayor claridad en la interpretación de las tendencias anuales de los parámetros calculados.

\section{RESULTADOS}

De los 21 inventarios realizados se ha determinado un total de 183 taxones (tab. 1; Anexo I) que pertenecen mayoritariamente a las rodofíceas (90 taxones: $49.4 \%$ ), siguiendo en importancia las feofíceas y clorofíceas (34 taxones cada grupo: $18.5 \%$ ) y en último lugar las cianofíceas (25 taxones: $13.6 \%$ ). Si se tiene en cuenta el número de taxones por inventario a lo largo del año, éste oscila entre 30 y 63. La dispersión de estos valores en un periodo estacional homogéneo es máxima entre noviembre y febrero (entre 30 y 63 taxones) y mínima en el periodo de julio a octubre (entre 31 y 40 taxones). Los valores de riqueza específica son mínimos en el periodo septiembre-octubre y máximos para enero-febrero (tablas 1 y 3 ).

Los taxones se estratifican verticalmente en cinco niveles que se relacionan a continuación junto a sus especies más características:
I: Estrato epífito superior: Feldmannia irregularis, Feldmannia caespitula, Antithamnion cruciatum, Ceramium rubrum, Dasya hutchinsiae, Calothrix confervicola y Catothrix aeruginea.

II: Estrato epífito medio: Titanoderma pustulatum y Titanoderma cystoseirae.

III: Estrato cespitoso basal: Valonia utricularis, Lophosiphonia reptabunda, Lophosiphonia cristata, Schottera nicaensis, Gelidium pusillum, Polysiphonia opaca, Polysiphoniaflocculosa, Gigartina acicularis, Corallina elongata, Cladophora laetevirens, Ceramium ciliatum var. robustum, Jania rubens, Padina pavonica, Chondria tenuissima, Anadyomene stellata (mediados de verano y otoño), Cladophoropsis modonensis (verano y otoño) y Laurencia microcladia (verano y otoño).

IV: Estrato incrustante basal: Spongites notarisii, Ralfsia verrucosa, Hildenbrandia canariensis, Pseudolithoderma adriaticum y Lithophyllum incrustans.

V: Subestrato perforante basal: Phaeophyla dendroides, Mastigocoleum testarum e Hyella caespitosa.

Los grupos ecológicos mejor representados en la comunidad definida por Laurencia papillosa se integran en los supergrupos de algas mediolitorales (FM $s l$ (sentido amplio): $\mathrm{FM}+\mathrm{RM}+\mathrm{RMM} 1+\mathrm{RMM} 2+\mathrm{RMI}+\mathrm{RMS}) \quad \mathrm{e}$ infralitorales fotófilas (PhI sl: $\mathrm{PhI}+\mathrm{PhIC}+\mathrm{PhIB}+\mathrm{PhIP}) . \quad \mathrm{La}$ relevancia fisionómica de ambos supergrupos evoluciona estacionalmente (fig. 1). Las especies pertenecientes a FM $s l$ son las de mayor relevancia desde enero hasta junio (especialmente en el intervalo mayo-junio). En el periodo julioagosto se ha incrementado la relevancia de las especies del supergrupo $\mathrm{PhI} s l$ que supera ligeramente la de las especies mediolitorales. Esta situación se mantendrá hasta diciembre.

Los supergrupos $\mathrm{PhI} s l$ y FM sl son netamente dominantes (dominancia cuantitativa) sobre el resto (SI $s l$ : esciáfilas 


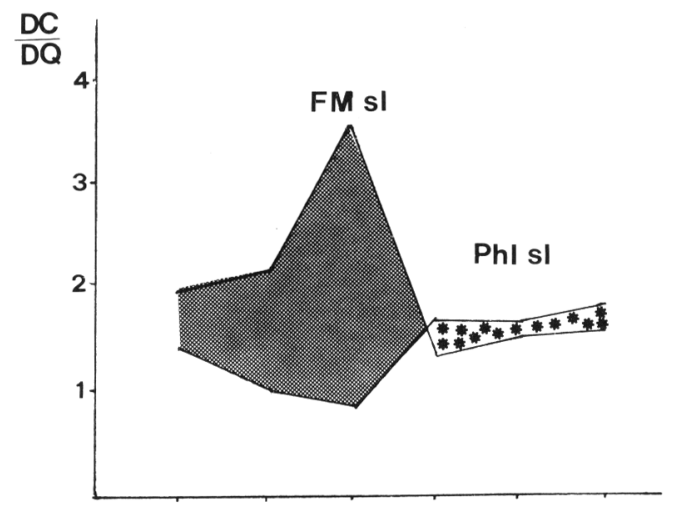

Figura 1: Evolución anual de la relevancia fisionómica (Cociente DC/DQ: dominancia cualitativa / dominancia cuantitativa). Las medidas se han realizado en periodos bimensuales desde Enero-Febrero a Noviembre-Diciembre. La trama granular corresponde al periodo de mayor relevancia de las algas mediolitorales y la estrellada al periodo de mayor relevancia de la algas infralitorales fotófilas. Annual variation of physionomical relevance (DC/DQ quotient: quantitative dominancelqualitative dominance). The granular welf shows a great dominance for intertidal taxa and starry walf for bottons photophilic taxa.

infralitorales; ISR: infralitorales de sustrato rocoso y ETN $s l$ : afinidades tionitrófilas en sentido amplio) cuyas dominancias cuantitativas son casi vestigiales (tab. 2).

La dominancia cuantitativa mínima de las especies infralitorales fotófilas se produce desde marzo hasta junio y se corresponde con el máximo en la dominancia cuantitativa de las especies mediolitorales. En este intervalo primaveral hay un ligero incremento de las especies esciáfilas (aunque nunca supera el $5.7 \%$ ) debido al mayor recubrimiento de Schottera nicaensis, Dasya ocellata, Cladophora lehmanniana y Callithamnion corymbosum.

En lo referente a la estructura de la comunidad a pequeña escala, los valores obtenidos para las áreas mínimas estructurales muestran una notable dispersión entre inventarios de un mismo periodo anual, especialmente entre los meses de marzo y junio, con valores medios entre 245 y $260 \mathrm{~cm}^{2}$, aunque ocasionalmente se precisan superficies de hasta $403 \mathrm{~cm}^{2}$ para tener una buena representación de la microestructura de la comunidad, por lo que este último valor debería de considerarse como referencia para sus muestreos estructurales. En el ciclo anual este parámetro experimenta amplias fluctuaciones estacionales (tab. 3), siendo mínima en el intervalo estival $\left(179 \mathrm{~cm}^{2}\right.$ por término medio) y máxima en los meses más fríos $\left(330 \mathrm{~cm}^{2}\right.$ por término medio). El área mínima cualitativa es, durante todo el año, menor a la estructural, no superando nunca superficies de $263 \mathrm{~cm}^{2}$, las cuales deben de considerarse idóneas para una buena representación de las especies de la comunidad.

La diversidad específica (Alfa-diversidad) sigue una evolución paralela en el ciclo anual al valor del área mínima cuantitativa. Es mínima en el periodo comprendido entre mayo

\begin{tabular}{lccccc}
\hline & PhI sl & FM sl & SCsl & ISR & ETN sl \\
\hline Enero-Febrero & $42.3 \pm 22$ & $40.7 \pm 14.2$ & $1.8 \pm 0.9$ & $5.3 \pm 7$ & $1.1 \pm 1.2$ \\
Marzo-Abril & $29.1 \pm 19$ & $50.3 \pm 14$ & $3.2 \pm 2.4$ & $5.8 \pm 9$ & $1.9 \pm 3.3$ \\
Mayo-Junio & $32.9 \pm 2.5$ & $56.5 \pm 15.3$ & $2.5 \pm 3.2$ & $3.6 \pm 1.7$ & $6.2 \pm 8.7$ \\
Julio-Agosto & $46.4 \pm 5.5$ & $39.8 \pm 10.7$ & $1.4 \pm 1.1$ & $3.2 \pm 4.4$ & $3.5 \pm 3.2$ \\
Septiembre-Octubre & $54.2 \pm 7.8$ & $37.2 \pm 15$ & $1 \pm 0.6$ & $0.6 \pm 0.8$ & $0.4 \pm 0.5$ \\
Noviembre-Diciembre & $57.2 \pm 7.8$ & $37.3 \pm 22$ & $1.3 \pm 1.5$ & $2.3 \pm 3.6$ & $0.3 \pm 0.5$ \\
\hline
\end{tabular}

Tabla 2: Evolución anual de la dominancia cuantitativa de grandes grupos ecológicos de algas. Se indican valores medios e intervalos de varianza. Annual variation of the quantitative dominance of the ecological groups is showed, with indication of the average dominance and the interval the variance. 


\begin{tabular}{lccccc}
\hline & $\begin{array}{c}\text { Area min. } \\
\text { cuantitativa }\end{array}$ & $\begin{array}{r}\text { Riqueza } \\
\text { florística }\end{array}$ & $\begin{array}{c}\text { Area min. } \\
\text { estructural }\end{array}$ & $\begin{array}{c}\text { Alfa } \\
\text { diversidad }\end{array}$ & $\begin{array}{c}\text { Beta } \\
\text { diversidad }\end{array}$ \\
\hline Enero-Febrero & $193.4 \pm 18.4$ & $50.8 \pm 12.5$ & $317 \pm 45.1$ & $4.6 \pm 0.9$ & $19.5 \pm 4.5$ \\
Marzo-Abril & $173.6 \pm 41$ & $45.4 \pm 3.1$ & $260.5 \pm 85.3$ & $4.3 \pm 1.1$ & $16.6 \pm 7.2$ \\
Mayo-Junio & $235 \pm 28.3$ & $49.2 \pm 2.2$ & $245.5 \pm 120.9$ & $3.9 \pm 1.7$ & $15.2 \pm 7.3$ \\
Julio-Agosto & $168.9 \pm 46.8$ & $36.3 \pm 3.8$ & $179.3 \pm 24.1$ & $3.4 \pm 0.6$ & $10.2 \pm 2$ \\
Septiembre-Octubre & $131.4 \pm 50$ & $33.7 \pm 2.5$ & $184.6 \pm 15.3$ & $3.6 \pm 0.1$ & $9.9 \pm 1.2$ \\
Noviembre-Diciembre & $96.2 \pm 61$ & $37.8 \pm 7.9$ & $330.3 \pm 73.3$ & $5.1 \pm 0.9$ & $21.9 \pm 7.2$ \\
\hline
\end{tabular}

Tabla 3. Evolución anual de varios parámetros estructurales. Se indican valores medios e intervalos de varianza. Annual variation of structural parameters, average values and varianza intervals are shown.

y octubre (meses más cálidos) y máxima en el periodo entre noviembre y febrero (meses más fríos). Fluctuaciones similares (tab. 3) se aprecian en la diversidad de motivo (Beta-diversidad).

\section{DISCUSIÓN}

La comunidad algal dominada por Laurencia papillosa muestra una notable diversidad de especies durante el año en las localidades muestreadas.

Las fluctuaciones periódicas de la dominancia cuantitativa de las algas medio e infralitorales en esta comunidad son atribuibles a factores intrínsecos como el ciclo de desarrollo de Laurencia papillosa que alcanza sus máximos recubrimientos en la transición primavera-verano y a factores extrínsecos como los cambios experimentados por el nivel medio del mar a consecuencia de los vientos dominantes, de tal modo que las «secas» (descenso del nivel del mar) son más frecuentes durante el invierno y primavera a causa de los vientos dominantes de componente Oeste en el Sureste ibérico (Soto, 1987) lo que dificulta la colonización mediolitoral de especies infralitorales. Por el contrario, en el periodo estival comienza una ligera regresión de Laurencia papillosa que coincide con vientos dominante de componente Este, generadores de acumulación de aguas en el litoral, elevan- do el nivel del mar (Soto, 1987) lo que favorece el establecimiento de especies infralitorales, esencialmente ubiquistas, en la zona mediolitoral inferior. Las especies esciáfilas, a pesar del porte elevado de Laurencia, no encuentran aquí su medio óptimo, de ahí su baja representación; no obstante, alcanzan un pequeño máximo coincidente con el mayor desarrollo de Laurencia papillosa que puede crear un ambiente poco iluminado. Esta ubicación de especies infralitorales esciáfilas en comunidades fotófilas con especie dominante de gran porte (generalmente Cystoseira mediterranea) ya ha sido comentada por otros autores en el Mediterráneo Occidental (Boudouresque, 1971 b; Boisset, 1987; Ballesteros, 1988), coincidiendo todos en la baja representación cuantitativa de este grupo de algas.

La comunidad presenta una alta equitabilidad si se atiende a los altos valores de alfa-diversidad, a diferencia de otras comunidades de igual ubicación litoral (Ballesteros, 1984) que es atribuible al alto hidrodinamismo general de la zona y a la baja inclinación de las plataformas en que se asienta la comunidad, que facilita la coexistencia de especies procedentes de otras comunidades circundantes así como el hecho de, en este caso, haber muestreado también el estrato incrustante. La beta-diversidad muestra una tendencia análoga a la observada en la alfadiversidad por lo que se infiere que la máxima 
estructuración y compartimentación de la comunidad coincide con su mayor equitabilidad. Este paralelismo puede deberse a la generación de espacios vacíos, debido a la adquisición por Laurencia papillosa de una ordenación de contagio cuando no está en un periodo óptimo de desarrollo (meses fríos), encauzando de esta forma la distribución espacial compartimentada de las especies que colonizan o viven en ese nivel litoral en esta época, o bien favoreciendo una mayor producción de estrato cespitoso basal (Niell, 1980). Este esquema dinámico es seguido también por otras comunidades intermareales atlánticas (Fernández et al., 1983).

\section{CONCLUSIÓN}

La vegetación dominada por Laurencia papillosa podría sustituir a la asociación Lithophylletum tortuosi J. Feldmann en el Sureste ibérico. Difiere de la anterior asociación esencialmente en la ausencia de Lithophyllum lichenoides, la presencia del molusco Dendropoma petraeum que tapiza el sustrato rocoso y presencia de un elevado elenco de taxones algales de afinidad cálida y distribución pantropical como Laurencia papillosa, Laurencia microcladia, Gracilaria armata, Wrangelia penicillata, Anadyomene stellata, Siphonocladus pusillus etc.

Este tipo de vegetación tampoco es encuadrable en la comunidad de Ceramietum ciliati Boudouresque, de similar ubicación litoral, debido al carácter de la especie definidora, Laurencia papillosa, perenne y fuertemente adherida al sustrato (rocaDendropoma-Spongites), el mayor tamaño de Laurencia, su adherencia al sustrato y su carácter perenne favorece el mantenimiento de un microhabitat más constante en el que proliferan especies, haciendo el sistema menos dependiente de eventos aperiódicos como «secas» y temporales.
Por lo comentado en párrafos anteriores, Laurencia papillosa podría considerarse como la especie definidora de una comunidad individualizada, propia de litorales en plataforma (aunque también colonice ocasionalmente litorales en pendiente) generalmente tapizados por vermétidos (principalmente Dendropoma petraeum $\_$del Sureste de España y probablemente de litorales similares de las áreas más cálidas del Mediterráneo Occidental.

\section{ANEXO I}

Relación de especies citadas en el texto y en las tablas.

Acetabularia acetabulum (L.) Silva

Amphiroa cryptarthrodia Zanardini

A. rigida Lamour.

Anadyomene stellata (Wulfen) C. Agardh

Anotrichum tenue (C. Agardh) Näg.

Antithamnion cruciatum (C. Agardh) Näg.

A. ogdeniae Abbot

Aphanocapsa littoralis Hansgirg

Audouinella daviesii (Dillwyn) Woelkerling

A. endophyta (Batters) Dixon

Audouinella moniliformis (Rosenvinge) Garbary

A. secundata (Kylin) Dixon

A. trifila (Buffham) Dixon

A. virgatula (Harv.) Dixon

Brachytrichia quojii (C. Agardh) Bornet et Flahault Bryopsis plumosa (Hudson) C. Agardh

Calothrix aeruginea Thur. ex Bornet et Flahault

C. confervicola (Roth) C. Agardh

C. crustacea Thur. ex Bornet et Flahault

Callithamnion corymbosum (Smith) Lyngb.

C. granulatum (Ducluz.,) C. Agardh

Ceramium ciliatum (Ellis) Ducluz. v. robustum (J. Agardh) Mazoyer

C. circinatum (Kütz.) J. Agardh

C. codii (Richards) Mazoyer

C. diaphanum (Lightf.) Roth

C. flaccidum (Kütz.) Ardiss.

C. rubrum (C. Agardh) v. barbatum (Kütz.) J. Agardh

C. rubrum C. Agardh v. tenue C. Agardh 
C. strictum Harv.

Cladophora coelothrix Kütz.

C. dalmatica Kütz.

C. laetevirens (Dillwyn) Kütz.

C. lehmanniana (Lindenb.) Kütz.

C. pellucida (Huds.) Kütz.

C. prolifera (Roth) Kütz.

C. sericea (Huds.) Kütz.

Cladophoropsis modonensis (Kütz.) Boergesen

Cladostephus hirsutus (L.) Prud'homme van Reine

Colpomenia sinuosa (Mertens ex Roth) Derbès et Solier

C. elongata Ellis et Solander

C. granifera Ellis et Solander

C. officinalis $\mathrm{L}$.

Crouania attenuata (C. Agardh) J. Agardh

Cystoseira compressa (Esper) Gerloff et Nizamuddin

C. mediterranea Sauv.

Chaetomorpha aerea (Dillwyn) Kütz.

C. gracilis Kütz.

Champia parvula (C. Agardh) Harv.

Chondria boryana (De Not.) De Toni

C. tenuissima (Goodenoug et Woodward) C. Agardh

Choreonema Thureti (Bornet) Schmitz

Chroococcus turgidus (Kütz.) Nägeli

Chroodactylon ornatum (C. Agardh) Basson

Dasya corymbifera J. Agardh

D. hutchinsiae Harv. in Hooker

D. ocellata (Grateloup) Harv. in Hooker

D. rigidula (Kütz.) Ardiss.

Derbesia tenuissima (De Not.) J. Feldm. et al.

Dermocarpa prasina (Reinsch) Bornet et Thur.

Dictyota dichotoma (Hudson) Lamour.

Dilophus fasciola (Roth) Howe

D. fasciola (Roth) v. repens (J. Agardh) J. Feldm.

D. spiralis (Mont.) Hamel

Dipterosiphonia rigens (Schousb.) Falkenb.

Ectocarpus siliculosus (Dillwyn) Lyngb. v. penicillatus C. Agardh

E. siliculosus (Dillwyn) Lyngb.

Enteromorpha compressa (L.) Grev.

Epicladia flustrae Reinke

Erythropeltis subintegra (Rosenvinge) Kornmann et Sanlihg

Erytrotrichia carnea (Dillwyn) J. Agardh

Falkenbergia rufolanosa (Harv.) Schmitz stadio

Feldmannia caespitula (J. Agardh) Knoep.-Pég.

F. caespitula (J.Agardh)Knoep.-Pég. v. lebelii (Aresch.)Hamel
F. irregularis (Kütz.) Hamel

F. padinae (Buffham) Hamel

Fosliella farinosa (Lamour.) Howe

Gastroclonium clavatum (Rothpletz) Ardiss.

Gelidiella lubrica (Kütz.) J. Feldm. et Hamel

G. pannosa (J. Feldm.) J. Feldm. et Hamel

Gelidium pusillum (Stack.) Le Jolis

Giffordia intermedia (Rosenvinge) Lund

G. ovata $(\mathrm{Kjellm}$.) Kylin

G. sandriana (Zanardini) Hamel

G. secunda (Kütz.) Batters

Gigartina acicularis (Roth) Lamour.

Gomphosphaeria aponina Kütz.

Gracilaria armata (C. Agardh) J. Agardh

Halimeda tuna (Ellis et Solander) Lamour.

Halopteris filicina (Grat.) Kütz.

H. scoparia (L.) Sauv.

Herposiphonia tenella (C. Agardh) Hambronn

H. tenella (C.Agardh) Ambronn v. secunda

(C.Agardh) Hollenberg

Hildenbrandia canariensis Boerg.

H. rubra (Sommerfelt) Menegh.

Hydrocoleum lyngbyaceum Kütz.

Hyella caespitosa Bornet et Flahault

Hypnea musciformis (Wulfen) Lamour.

Isactis plana Thur.

Janczewskia verrucaeformis Solms

Jania adhaerens Lamour.

J. corniculata (L.) Lamour.

J. rubens (L.) Lamour.

Laurencia microcladia Kütz.

L. obtusa (Huds.) Lam. v. pyramidata J. Agardh

L. obtusa (Huds.) Lamour.

L. papillosa (C. Agardh) Grev.

L. pinnatifida (Huds.) Lamour.

Lophosiphonia cristata Falkenb.

L. reptabunda (Suhr in Kütz.) Kylin

L. subadunca (Kütz.) Falkenb.

Lyngbya confervoides $\mathrm{C}$. Agardh

L. epiphytica Hieron

L. mayuscula Harv. in Hooker

L. meneghiniana Gomont

L. sordida (Zanardini) Gomont

Mastigocoleus testarum Lagerheim ex Bornet et Flahault

Melobesia membranacea (Esper) Lamour.

Monosporus pedicellatus (Smith) Solier in Castagne Myrionema strangulans Grev.

Nemalion helminthoides (Velley in Withering) Batters 
Nemoderma tingitanum Schousb. ex Bornet

Ochlochaete histrix Thwaites ex Harv.

Oscillatoria bonnemaisonei Crouan frat.

$O$. corallinae Gomont

Padina pavonica (L.) Thivy

Pedobesia Lamourouxii (J. Agardh) J. Feldm.et al.

Peyssonnelia dubyi Cruan frat.

P. rubra (Greville) J. Agardh

Phaeophila dendroides (Crouan frat.) Batters

$P$. viridis (Reinke) Parke et Burrow

$P$. wittrockii (Wille) Nielsen

Phormidium spongeliae (Schulze) Gomont

Pilinia rimosa Kütz.

Pneophyllum lejolisii (Rosanoff) Chamberlain

Polyphysa parvula (Solms) Schnetter et Bula-Meyer

Polysiphonia breviarticulata (C. Agardh) Zanardini

P. flocculosa (C. Agardh) Kütz.

P. fruticulosa (Wulfen) Sprengel

P. opaca (C. Agardh) Morris et De Not.

P. tenerrima Kütz.

$P$. violacea (Roth) Sprengel

Porphyra umbilicalis (L.) J. Agardh

Pringsheimiella scutata (Reinke) Marchewianka

Pseudochlorodesmis furcellata (Zanardini)

Boergesen

Pseudolithoderma adriaticum (Hauk) Verlaque

Pterosiphonia thuyoides (Harv. in Mckay) Schmitz

Ralfsia verrucosa (Areschoug) J. Agardh

Rhizoclonium riparium Harv.

Rivularia atra Roth

$R$. bullata (Poiret) Berkeley ex Bornet et Flahault

$R$. mesenterica Thur.

$R$. polyotis (J. Agardh) Bornet et Flahault

Scytosiphon lomentaria (Lyngb.) Link

Schottera nicaensis (Lamour. es Duby) Guiry et Hollenberg

Siphonocladus pusillus (Kütz.) Hauck

Sphacelaria cirrosa (Roth) C. Agardh

S. furcigera Kütz.

S. rigidula Kütz.

S. tribuloides Menegh.

Spongites notarisii (Dufour) Athanasiadis

Spyridia filamentosa (Wulfen) Harv. in Hooker

Stylonema alsidii (Zanardini) Drew

S. cornu-cervi Reinsch

Symphyocarpus strangulans Rosenvinge

Symploca hypnoides Kütz.

Taenioma nanum (Kütz.) Papenfuss

Titanoderma corallinae (Crouan frat.) Woelkerling et. al.
T. cystoseirae (Hauck) Woelkerling, Chamberlain et Silva

T. hapalidiodes (Crouan frat.) Price, John et Lawson

T. pustulatum (Lamour.) Näg.

Ulva rigida $\mathrm{C}$. Agardh

Valonia utricularis (Roth) C. Agardh

Wrangelia penicillata $\mathrm{C}$. Agardh

\section{BIBLIOGRAFÍA}

BALLESTEROS, E. -1984-Els vegetals i la zonació litoral. Espècies, comunitats i factors que influeixen en la seva distribució. Tesis Doctoral. Universidad de Barcelona. 587 pp.

BALLESTEROS, E. -1986- Métodos de análisis estructural de comunidades naturales, en particular del fitobentos. Oecologia aquatica, 8: 113-131.

BALLESTEROS, E. -1988- Estructura y dinámica de la comunidad de Cystoseira mediterranea Sauvageau en el Mediterráneo noroccidental. Inv. Pesq., 52(3): 313-334.

BALLESTEROS, E. y J. ROMERO -1982- Catálogo de las algas bentónicas (con exclusión de las diatomeas) de las costas catalanas. Collect. Bot., 13: 723-765.

BARCELÓ, M.C.-1987- Estudi de les algues marines del Pais valenciá. Tesis Doctoral. Universidad de Barcelona. 487 pp.

BOISSET, F.-1987- Estudio del fitobentos esciáfilo infralitoral de sustratos duros en el litoral valenciano (España): Flora y vegetación. Tesis Doctoral. Universidad de Valencia. 387 pp.

BOUDOURESQUE, CH.F.-1971a- Méthodes d'étude qualitative et quantitative du benthos (en particulier du phytobenthos). Téthys, 3(1): 79-104.

BOUDOURESQUE, CH.F. -1971b- Recherches de bionomie analytique, structurale et expérimentale sur les peuplements benthiques sciaphiles de Méditerranée occidentale (Fractión algale): La sous-strate sciaphile des peuplements des grandes Cystoseira de mode battu. Bull. Mus. Hist. Nat. Marseille, 31: 141151.

BOUDOURESQUE, CH.F. - 1974- Aire minima et peuplements algaux marins. Soc. Phycol. Fr. Bull., 19: 141-147.

BOUDOURESQUE, CH.F. -1984- Grupes 
écologiques d'algues marines et phytocénoses benthiques en Méditerranée Nord-occidentale: une revue. Giorn. Bot. Ital., 118(1-2) suppl., 2: 7-42.

BOUDOURESQUE, CH.F. \& M. PERRET -1987A check-list of the benthic marine algae of Corsica. GIS posidonia publ., Marseille, 121 pp.

CINELLI, F., E. FRESI, E. IDATO \& I. MAZZELLA -1977- L'aire minima du phytobenthos dans un peuplement à Cystoseira mediterranea de l'ile d'Ischia (Golfe de Naples). Rapp. comm. int. Médit., 24 (4): 113-115.

CONDE, F. -1984- Catálogo de las algas macrobentónicas marinas de Málaga. Acta Bot. Malacitana, 9: 47-78.

CONDE, F. y J. SOTO -1986- Notas corológicas del macrofitobentos de Andalucía (España). Acta Bot. Malacitana, 11: 9-16.

CONDE, F. y J. SOTO -1987- Nuevas contribuciones al estudio de la vegetación bentónica marina del litoral granadino. Act. VI. Simp. Nac. Bot. Cript., 1: 35-42.

COPPEJANS, E. -1980- Phytosociological studies on Mediterranean algal vegetation: Rocky surfaces of the photophilic infralitoral zone. In The Shore environment. Vol. 2: Ecosystems: 371-393. Academic Press. London.

FERNÁNDEZ, C., F.X. NIELL y R. ANADON 1983- Comparación de dos comunidades de horizontes intermareales con abundancia de Bifurcaria bifurcata Ros. en las costas N y NO de España. Inv. Pesq., 47(3): 435-455.

GIACCONE, G., P. COLONNA, C. GRAZIANO, A. MANNINO, E. TORNATORE, M. CORMACI, G. FURNARI \& B. SCAMMACA 1985- Revisione della flora marina di Sicilia e isole minori. Boll. Acc. Gioenia Sci. Nat., 18: 537-781.

MAYHOUB, H. -1976-Recherches sur la végetation marine de la côte syrienne. Étude experimentale sur la morphogenèse et le développement de quelques espèces peu connues. Thèse. Université de Caen. 286 pp.

NIELL, F.X. -1980- Efectos de la destrucción del estrato de Himantalia elongata en la vegetación cespitosa del sistema intermareal de la Ría de Vigo. Inv. Pesq., 44(2): 253-263.

RIBERA, M.A. y A. GÓMEZ GARRETA -1984Catálogo de la flora bentónica de las Islas Ba- leares, I (Rhodophyceae). Collect. Bot., 15: 377-506.

SEOANE, J. -1965- Estudios sobre las algas bentónicas de la costa Sur de la Península Ibérica (Litoral de Cádiz). Inv. Pesq., 29: 3-216.

SOTO, J. -1987- Estudio florístico, corológico, autoecológico y sinecológico de las algas bentónicas marinas del Sureste de la Península Ibérica. Tesis Doctoral. Universidad de Málaga. $507 \mathrm{pp}$

SOTO, J. -1991- Datos sobre la flora y corología algal en el Mediterráneo de Andalucía (España): Litoral de Granada. Anales de Biología, 17 (Biología Vegetal, 6): 105-108. Secr. de Publ. Univ. de Murcia.

TERRADAS, J. (ed.) -1989- Història Natural del Països Catalans. Sistemes Naturals. Enciclopedia catalana. Vol. 14. Barcelona. 500 pp.

VARO, J., J. RAMIREZ y J. RENTERIA -1979Estudio de la vegetación bentónica del litoral de granadino. Acta Bot. Malacitana, 5: 79-98.

Aceptado para su publicación en Mayo de 1993

Dirección de los autores. J. Soto Moreno: Departamento de Biología Vegetal. Facultad de Biología. Universidad de Murcia. 30100 Murcia. España. F. Conde: Dpto. Biología Vegetal. Fac. de Ciencias. Universidad de Málaga. 29080 Málaga. 


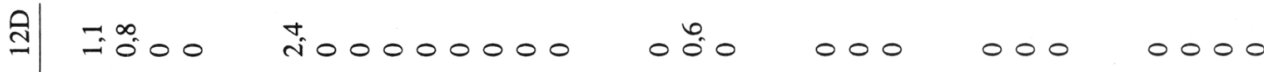

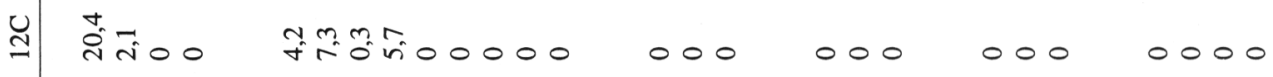

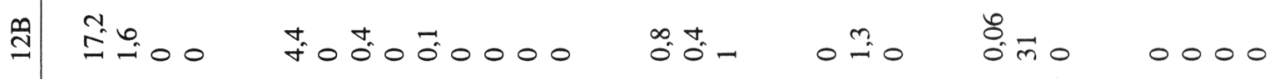

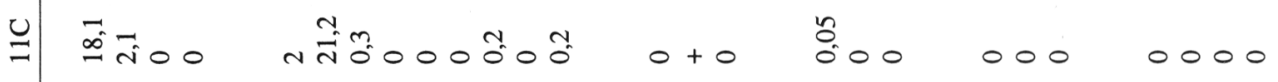

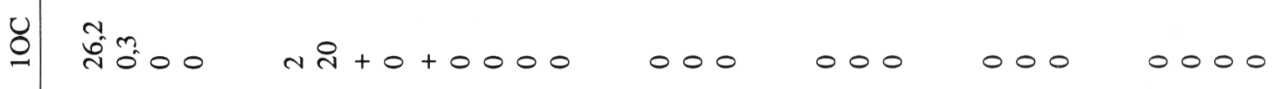

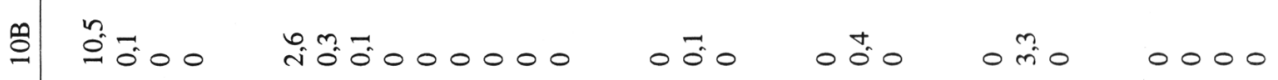

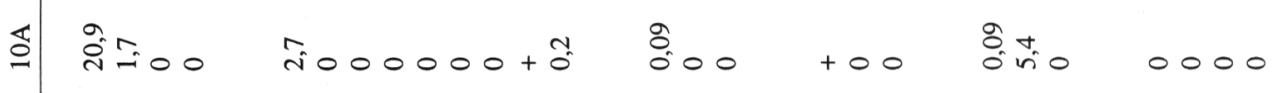

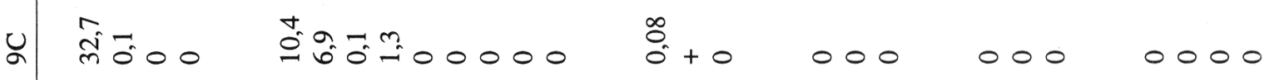

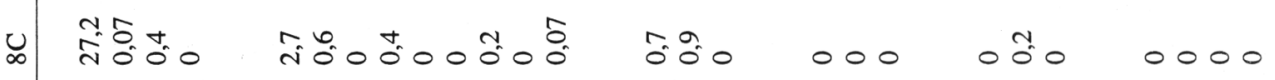

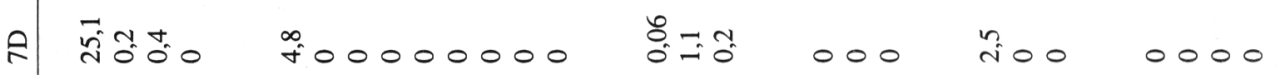

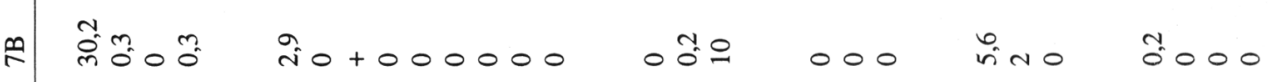

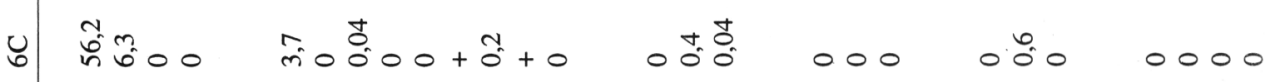

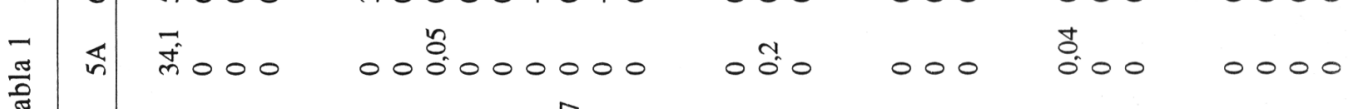
लि

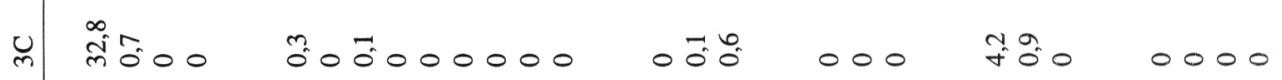

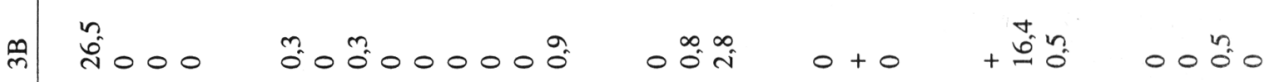

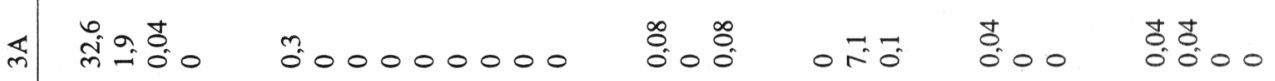

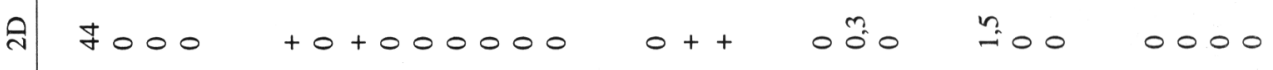

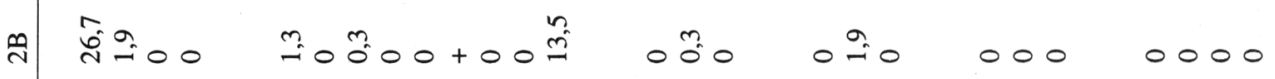

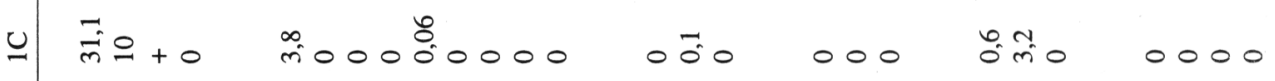

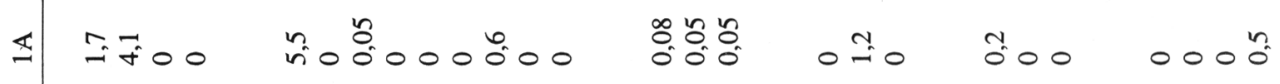

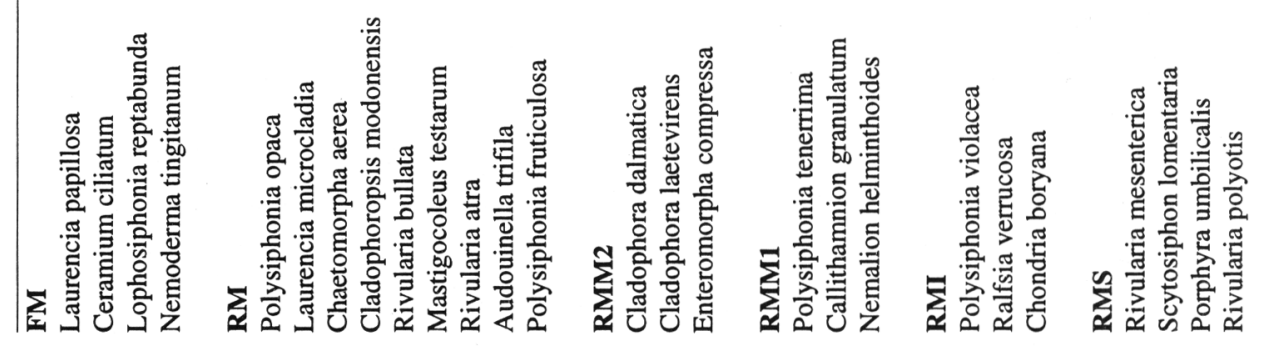




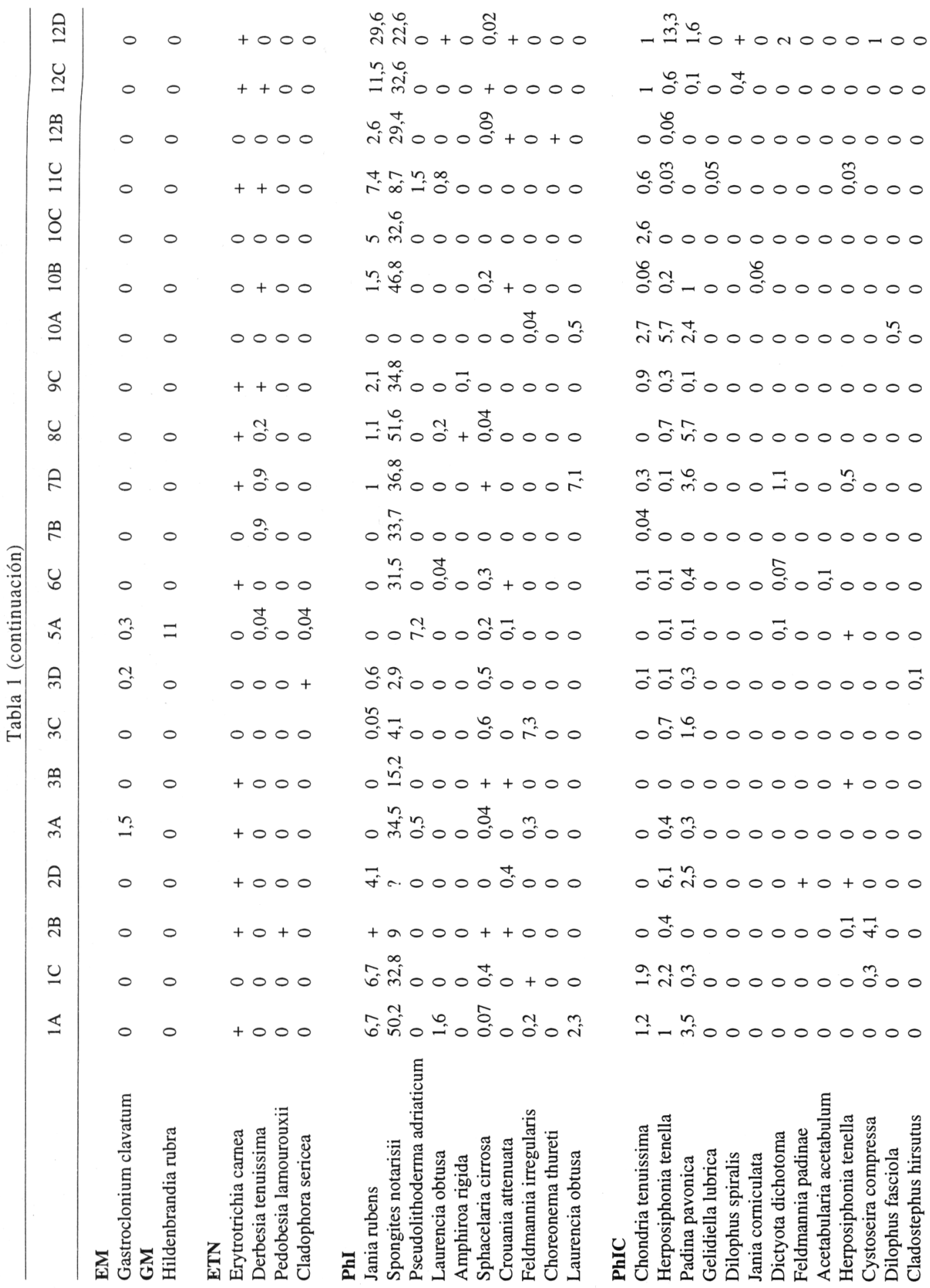




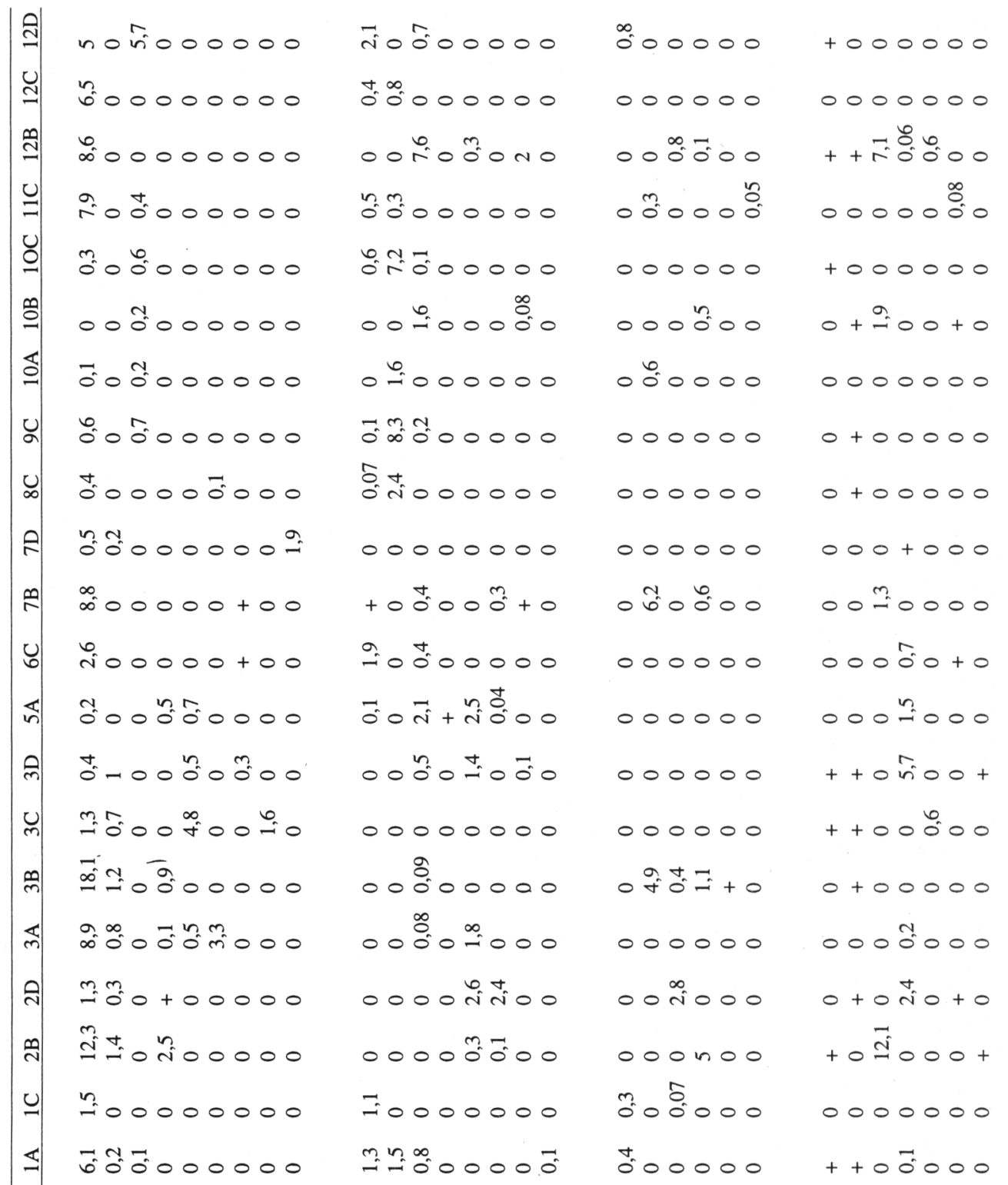
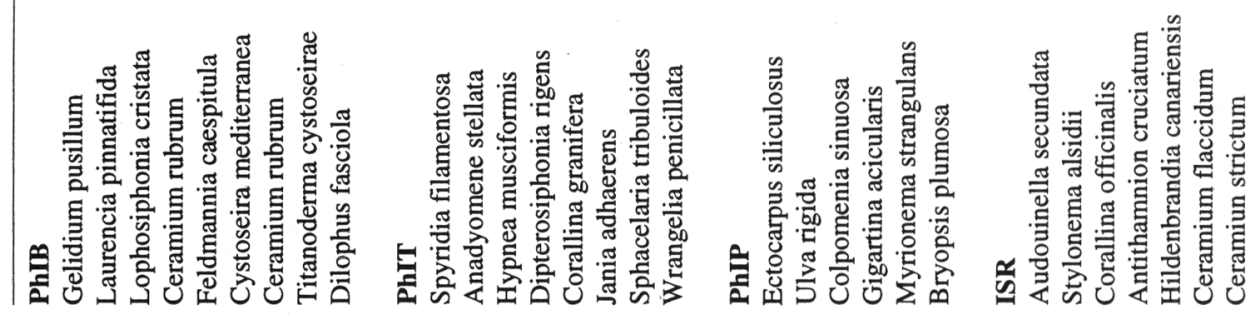


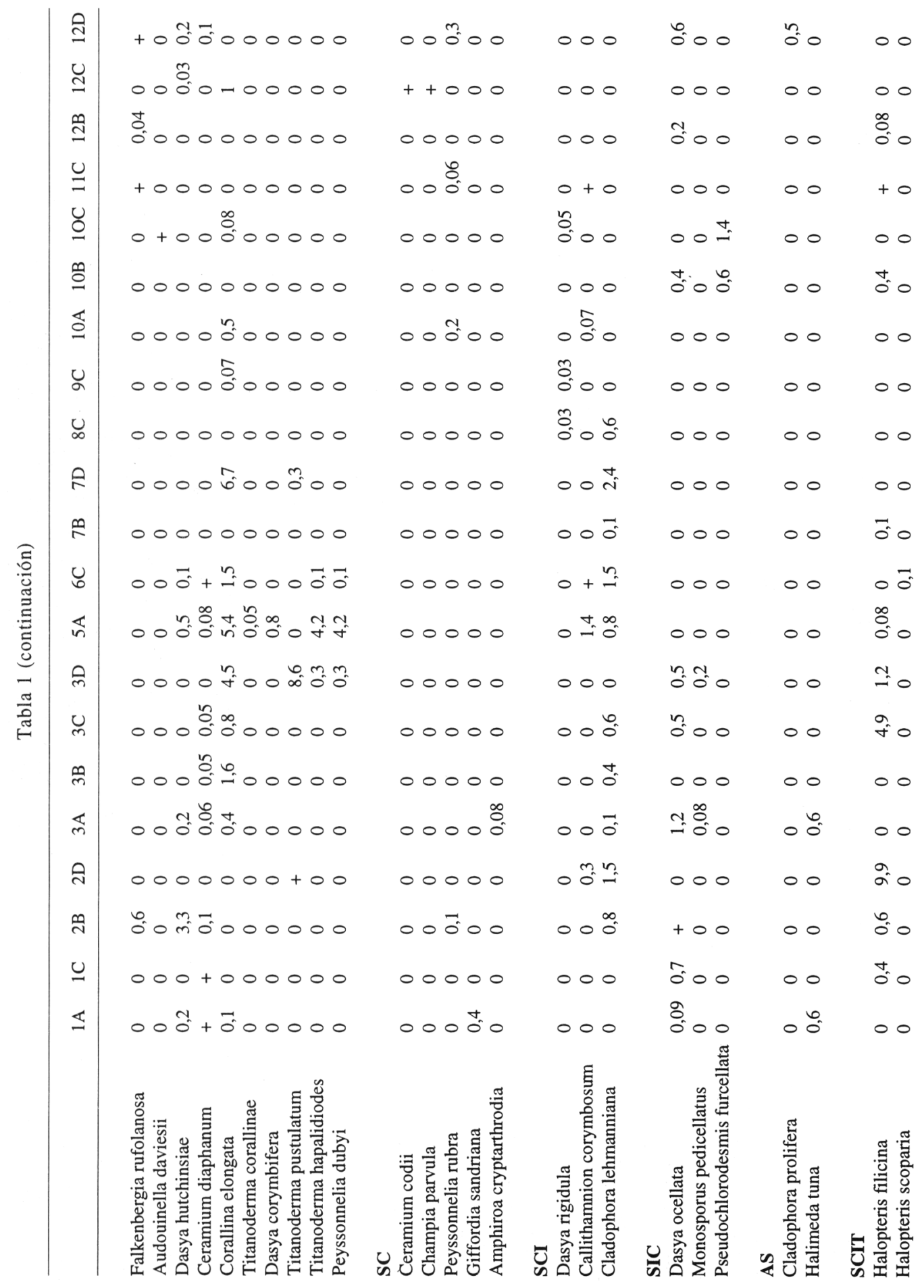




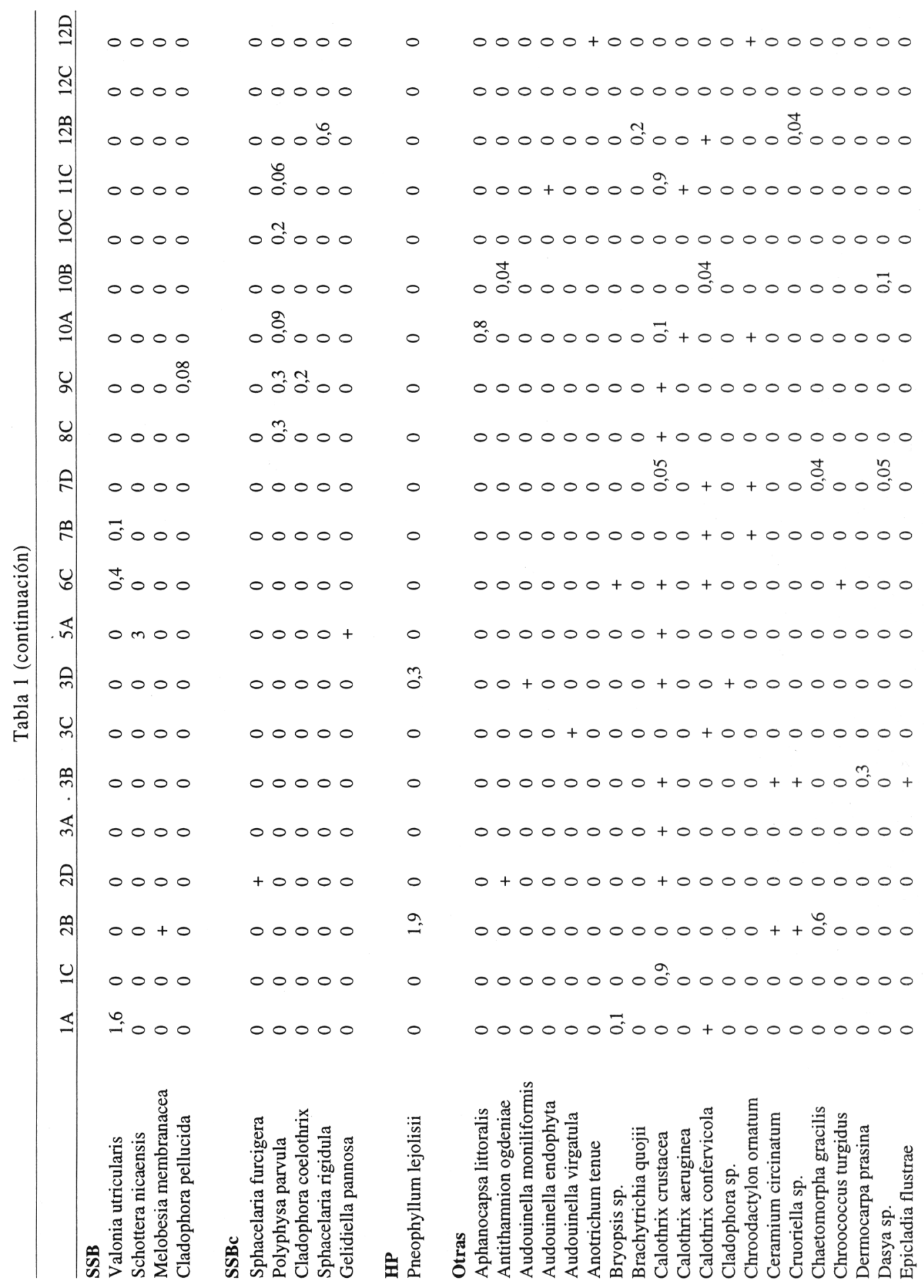




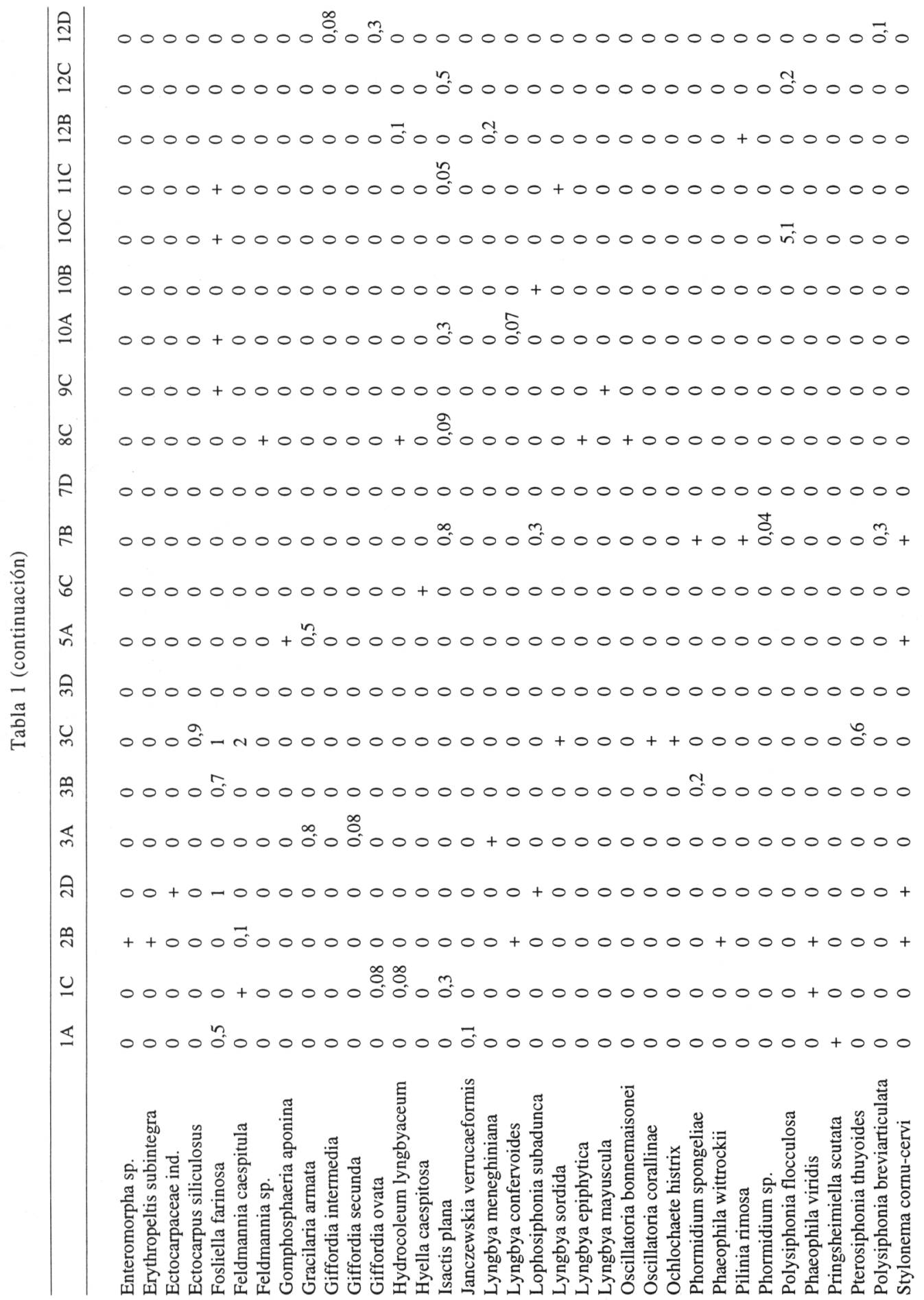



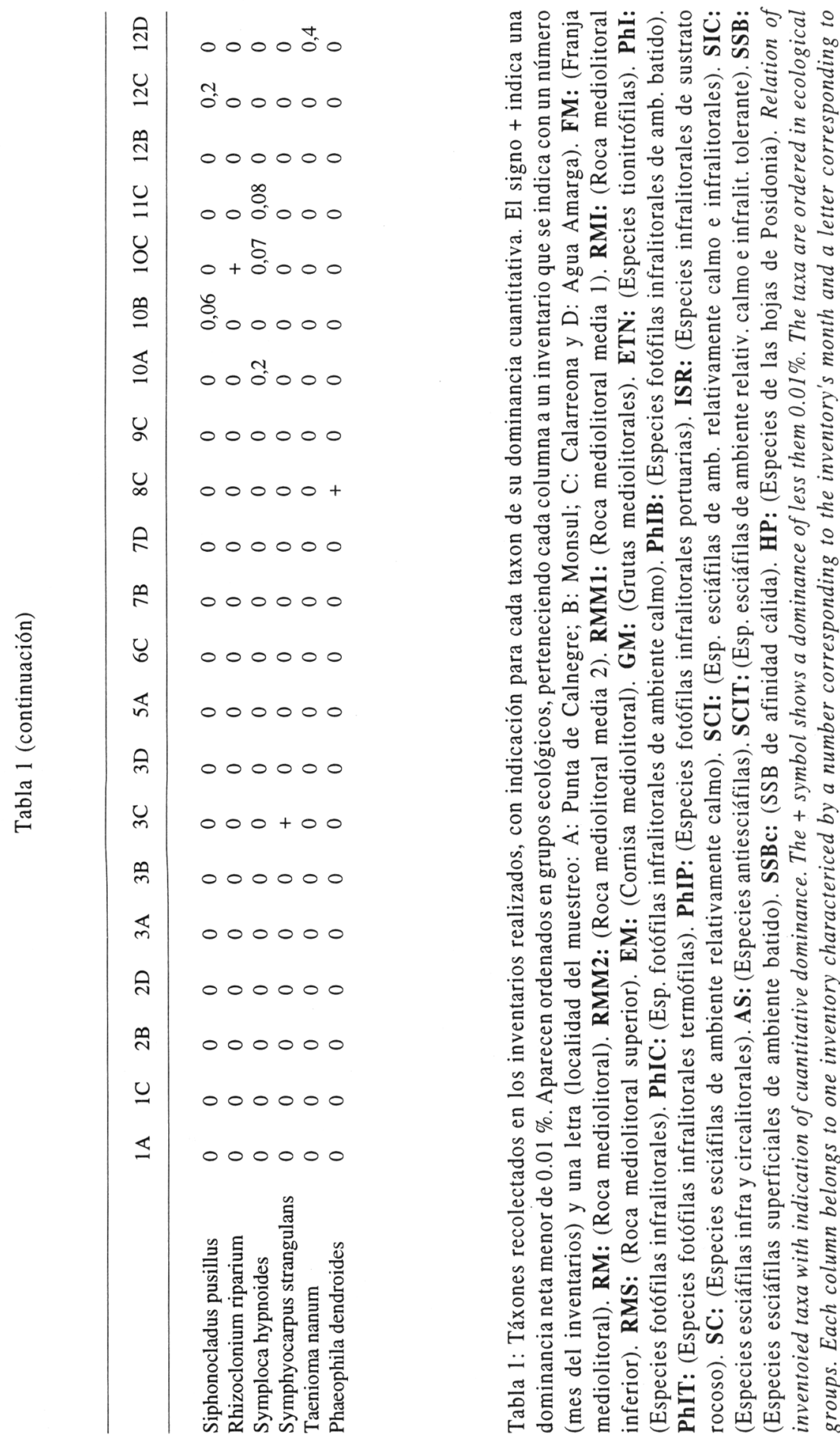
क

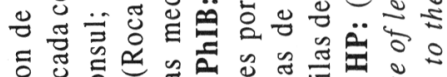
ప

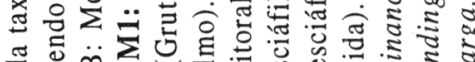

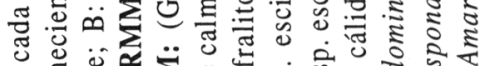

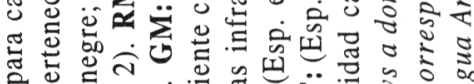
ป

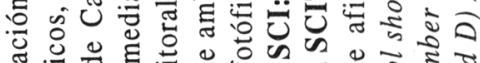

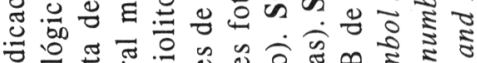

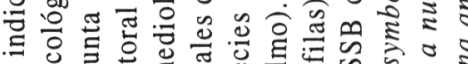
б

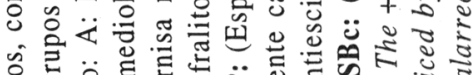

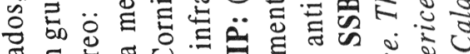

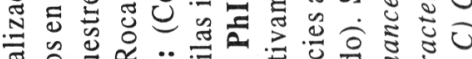

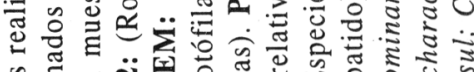

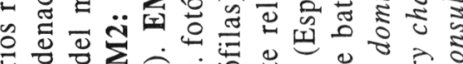

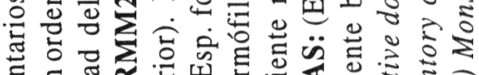

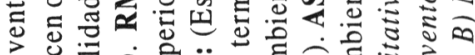

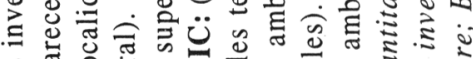

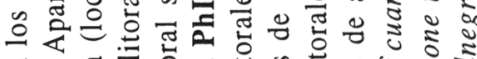

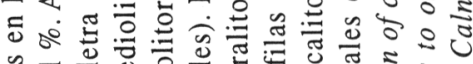

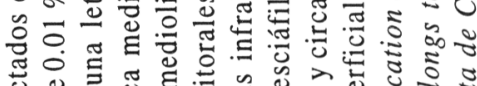

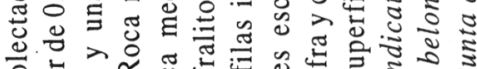

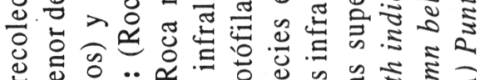

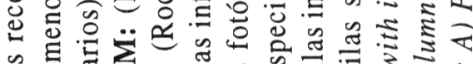

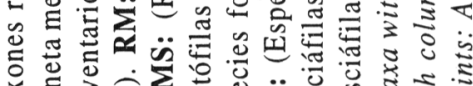

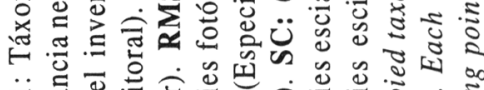

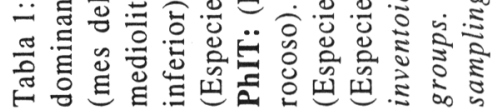

